\title{
Obra viva: estudio de la construcción y contexto de uso de una embarcación naufragada en el Río de la Plata en el siglo XIX
}

\section{(1) Ana Castelli*}

\author{
Fecha de defensa: 17 de Agosto de 2017 \\ Directora: Dra. Dolores Elkin \\ Co-director: Arq. Cristian Murray \\ Jurados: Dres. Virginia Pineau y Marcelo \\ Weissel
}

\section{Introducción al objeto de estudio}

En noviembre de 1998 se realizaron las excavaciones necesarias para los cimientos del Hotel Hilton ubicado en el dique 3 de Puerto Madero, Ciudad Autónoma de Buenos Aires. En este contexto se hallaron los restos de una embarcación de madera y otros materiales que fueron severamente dañados por la maquinaria pesada usada en la obra. Aunque ya descontextualizado, parte del conjunto pudo rescatarse y trasladarse al Instituto Nacional de Antropología y Pensamiento Latinoamericano, donde quedaron a cargo del Programa de Arqueología Subacuática. En el marco de las tareas de rescate (Weissel, 1998), se realizó un inventario de los materiales recuperados en el sitio. Estudios sedimentológicos permitieron determinar que los restos se encontraban en el lecho fluvial original, por debajo del relleno que se colocó para ganar terreno al río al construir Puerto Madero a fines del siglo XIX. Las obras en el dique tres, por ende, constituyen un terminus ante quem del naufragio: el año 1892 (fecha de inauguración del dique). A partir de los materiales asociados se estimó que la embarcación correspondería al siglo XIX. No obstante, el momento y lugar precisos de su construcción se desconocían al momento de comenzar este estudio, así como la función que esta cumplió en la navegación vinculada al puerto de Buenos Aires. Una primera observación de las piezas estructurales por parte de Cristian Murray, especialista en arquitectura naval, llevó a considerar que era de porte pequeño e incapaz de cruzar océanos. Embarcaciones de estas características han sido escasamente analizadas en Argentina desde una perspectiva arqueológica y cuentan con poca visibilidad en las fuentes documentales. Este caso presentó una oportunidad excepcional para estudiar aspectos poco conocidos de la navegación y la tecnología náutica en la cuenca del Plata.

El material de estudio consistió en los restos de la estructura de la embarcación referida, que comprende

* Instituto Nacional de Antropología y Pensamiento Latinoamericano (INAPL). 3 de Febrero 1378 (CP C1426BJN) Ciudad Autónoma de Buenos Aires, Argentina. E-mail: anitacastelli.leg@gmail.com maderamen y clavazón metálica (Figura 1). Si bien son piezas fragmentarias y desarticuladas, sus características permitieron estudiarlas de modo sistemático.

\section{Objetivos de investigación}

Los principales objetivos de este trabajo fueron caracterizar el diseño y la tecnología empleados en la construcción de la embarcación y precisar el contexto temporal y espacial de su fabricación y uso. Con ese fin, se buscó determinar sus dimensiones generales y características principales (forma, tipo, sistema de propulsión), reconocer las principales técnicas aplicadas en su manufactura, evaluar asociaciones entre la manufactura y la industria naval histórica en el área de estudio y analizar la posible función de la embarcación en el contexto de la navegación regional.

\section{Marco teórico}

Este trabajo se enmarcó en la Arqueología marítima histórica, que estudia el vínculo del ser humano con diversos entornos acuáticos y la Arqueología náutica, que se engloba en aquella y trata específicamente con las embarcaciones y la navegación. Una embarcación es un artefacto complejo, cuya construcción conlleva un gran número de decisiones de las que su diseño y manufactura pueden dar cuenta. Están insertas en cadenas de aprovisionamiento de materias primas, manufactura, uso, mantenimiento y descarte (sensu Schiffer, 2008), sujetas a constreñimientos que condicionan su diseño (Adams, 2001) y si bien son concebidas de manera ideal para un fin determinado, su realización material no siempre se ajusta a esos parámetros ideales (Pomey, 2011). Por estos motivos, las embarcaciones son artefactos que reflejan en múltiples niveles a la sociedad en el seno de la cual fueron gestadas. El estudio de la estructura de los naufragios, por lo tanto, debe trascender la mera descripción morfológica.

\section{Metodología}

El material se relevó detalladamente con medios fotográficos, gráficos y escritos, utilizando una metodología diseñada especialmente para dar cuenta de factores potencialmente diagnósticos y permitir la comparación de variables. Se realizaron tres tipos de análisis: arquitectónico, de carpintería y de materias primas. Los resultados derivados de los análisis se discutieron a la luz de fuentes documentales y tomando en cuenta los datos 


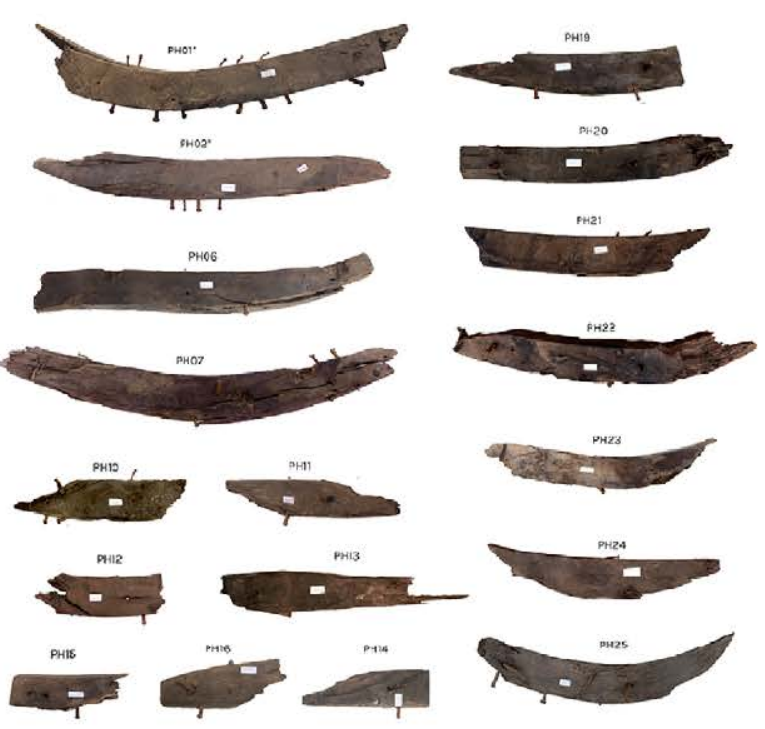

Fragmentos de LigAZÓN

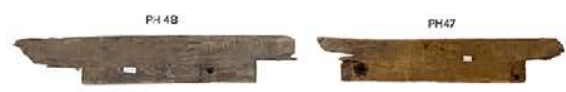

DURMIENTES

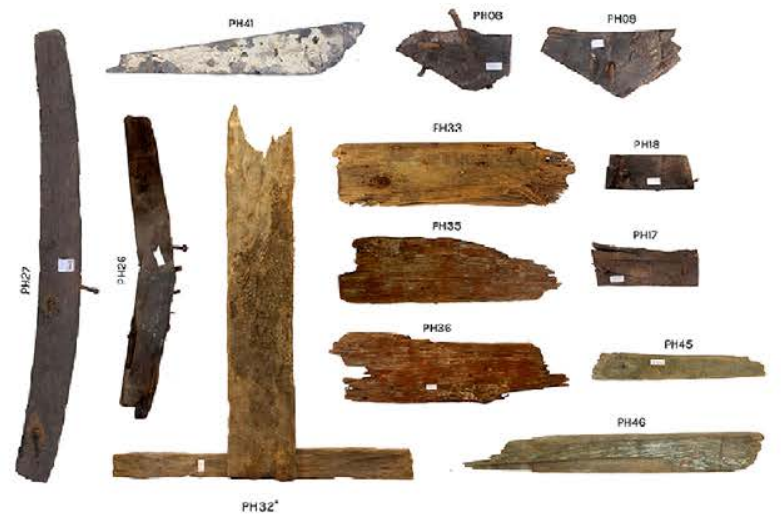

PiezAs no identificadas

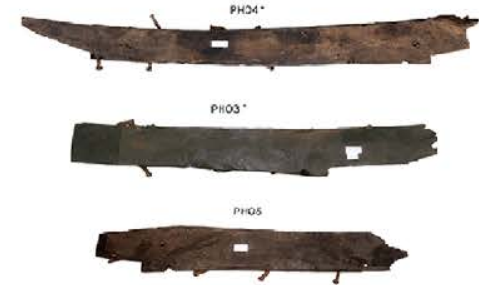

VARENGAS Y GENOLES
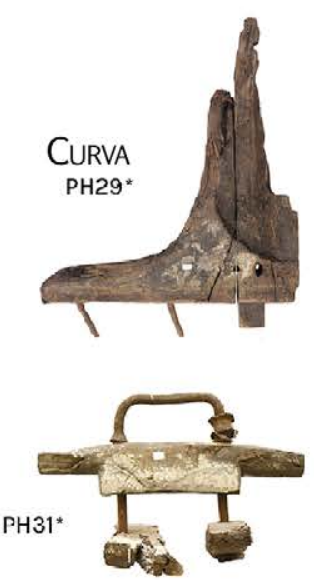

Bita de Amąre Pala de Timón

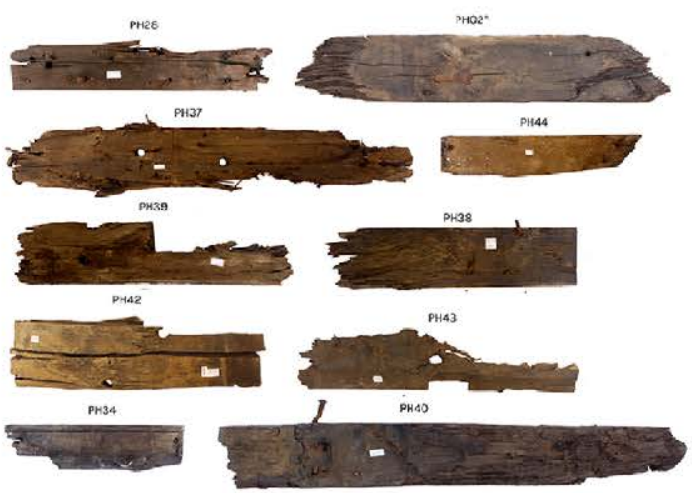

TABLAZÓN

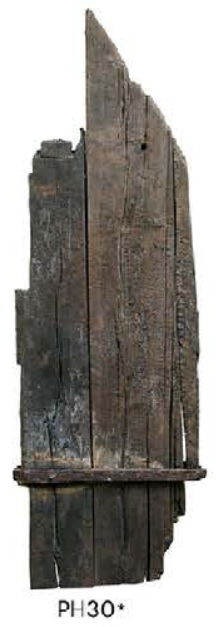

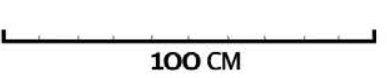

Figura 1. Piezas estructurales estudiadas.

recabados de fuentes bibliográficas acerca de la industria naval de tiempos históricos en el Río de la Plata.

En el análisis arquitectónico se identificaron las piezas en base a manuales constructivos y diccionarios náuticos, además de considerar otros trabajos arqueológicos pertinentes. Se distinguieron fragmentos de cuaderna (ligazones, genoles, varengas), de tablazón y de otros elementos (durmientes, pala de timón, curva y bita de amarre) (Figura 1). Con los remanentes de cuadernas pudo 
además estudiarse la ubicación relativa de las piezas en la estructura, el método de montaje, las curvaturas y posibles secciones y los tipos y método de clavazón.

El análisis de carpintería fue diseñado para reconocer técnicas constructivas específicas utilizadas y localizar patrones en el uso y aplicación de distintos materiales. Para esto, se clasificaron y cuantificaron las evidencias de uso de herramientas, adherencias y coberturas y su presencia en diferentes sectores de las piezas.

Para el análisis de materias primas se sumaron trabajos de caracterización botánica y metalúrgica, sobre el maderamen y la clavazón. La información botánica fue realizada previo al desarrollo de esta tesis, por el Dr. Ignacio Mundo ${ }^{1}$. Por otro lado, los análisis arqueometalúrgicos fueron realizados por la autora, en el ámbito del Grupo de Arqueometalurgia de la Facultad de Ingeniería de la Universidad de Buenos Aires y bajo la tutela de su director, el Ing. Horacio de Rosa. El conocimiento derivado de los análisis botánicos fue aplicado para reconocer la procedencia geográfica de las materias primas, mientras que el estudio metalúrgico buscó, a través de la caracterización tecnológica de los elementos de clavazón, precisar el contexto temporal en que fue construido el barco.

\section{Resultados}

Los análisis revelaron que la embarcación fue concebida para responder a las necesidades de la navegación en la cuenca del Plata, donde las bajas profundidades son un condicionante sin par, pero no resultaba adecuada para navegar en altamar. Fue diseñada para perdurar y posiblemente era apta para cargas y trabajos pesados. La forma del fondo del casco es casi plana, lo cual se deduce del ángulo con que las varengas parten de la quilla. Posiblemente poseyera propulsión a vela. Algunos elementos del conjunto sugieren la presencia de un mástil y una botavara y no se conservó evidencia alguna de propulsión a vapor o motores. Se desconocen las características de la quilla y las estructuras de popa y proa. Sin embargo, el estudio arquitectónico permitió una aproximación a las dimensiones de la embarcación: la reconstrucción gráfica de la sección media del casco sugiere una manga máxima de alrededor de tres metros (Figura 2) y la comparación con casos arqueológicos e históricos sugiere una eslora de entre nueve y 15 metros y un puntal inferior a dos metros. En la colección se reconocieron durmientes de bao y tablas asociadas a una bita que posiblemente correspondan a la estructura de una cubierta o media cubierta, que era fijada con cabillas de madera. Todas estas características son coherentes con un tipo de embarcación

${ }^{1}$ Instituto Argentino de Nivología, Glaciología y Ciencias Ambientales, Consejo Nacional de Investigaciones Científicas y Técnicas (CONICET) Mendoza. frecuente en las fuentes históricas e iconográficas del siglo XIX: la balandra, destinada al comercio de cabotaje y al trasbordo de cargamento y pasajeros. Debemos considerar que una embarcación de estas características pudo haber cumplido numerosas funciones y haber sido acondicionada a lo largo de su vida útil. En lo que respecta a su utilización, las dimensiones y el diseño de su casco; su sistema de propulsión, con un mínimo de una vela y una pala de timón recta; y la información derivada de fuentes históricas, señalan que una embarcación de este tipo podría haber navegado por el Río de la Plata y otras zonas transitables de la cuenca, e incluso realizado viajes cortos de cabotaje, por ejemplo, hasta el sur de Brasil o el sur de la provincia de Buenos Aires.

En base a los estudios botánicos, las maderas llegaban desde la región chaqueña y la selva paranaense y eran de buena calidad y gran dureza. Por otro lado, los elementos de fijación pudieron haber llegado en cargamentos desde el exterior, posiblemente Inglaterra o Estados Unidos; o bien haber sido obtenidos mediante barras de hierro trabajadas por herreros locales.

Con respecto a los constructores (carpinteros, calafates, herreros, etc.), la notoria falta de tratados constructivos e incluso relatos acerca de ellos en el registro documental local, señala que es posible que sus conocimientos se transmitieran a través de tradición oral. Los carpinteros de ribera definieron la forma y dimensiones que las piezas debían tener para convertirse en el barco previamente concebido, e invirtieron considerable esfuerzo en el trabajo de la madera. Utilizaron sierras y azuelas y mejoraron las terminaciones con herramientas como cepillos. Si bien existen algunos calados decorativos, todos son sencillos. El estudio reveló que los carpinteros construyeron las cuadernas en posición horizontal, uniendo las ligazones dobles con pernos de hierro y sin uniones entre cabezales, posiblemente incorporando marcas en las maderas que les sirvieron de guía. Utilizaron taladros manuales para realizar orificios de diámetro levemente menor al de los pernos, que eran forzados mediante martillado. Estos pernos no atravesaban las piezas por completo y las mantenían unidas sólo por medio de fricción, sin arandelas o cabezas. Las cuadernas se montaron sobre la quilla desde la cuaderna maestra hacia popa y hacia proa, con un espaciado de alrededor de $36 \mathrm{~cm}$. Una vez montada la estructura, los carpinteros clavaron las tablas de forro externas e internas, con clavos forjados grandes para las primeras y clavos de máquina más pequeños para las segundas. Clavazón adicional servía de refuerzo. Posiblemente las costuras entre las tablas fueron impermeabilizadas con estopa y brea, alquitrán, o alguna mezcla semejante, entre las uniones. Finalmente, algunas piezas visibles fueron pintadas, como la bita de amarre. Otras piezas presentaban masillas claras y otros colores de pintura, incluyendo 


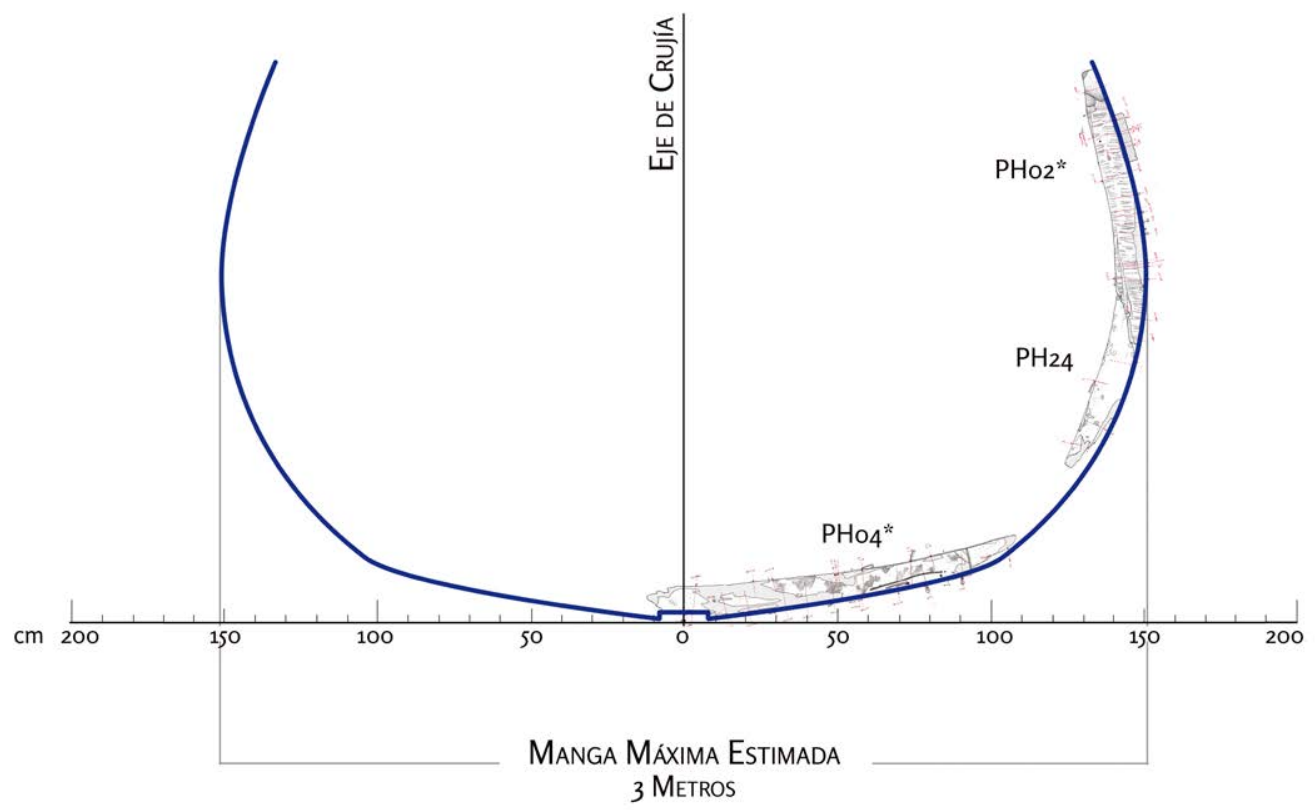

Figura 2. Proyección de curvas hacia el sector medio de la embarcación, que indica un fondo casi plano y una manga de alrededor de 3 metros.

algunas tablas de forro. Las cuadernas, por otro lado, no mostraban ningún tipo de pintura.

Gracias al estudio arquitectónico y la aplicación de técnicas arqueométricas, se ha podido precisar el período en que la embarcación fue manufacturada y utilizada. Los clavos de máquina se distribuyeron a partir de 1830, antes de lo cual no estaban disponibles en el país. Su recurrencia en las piezas, especialmente las cuadernas, sugiere que no corresponden a reparaciones sino a la construcción original. Por este motivo, el barco probablemente fue construido luego de 1830 y, como se ha mencionado, debió hundirse necesariamente antes de 1892 . Es probable que haya estado en funcionamiento hacia mediados del siglo XIX y haya sufrido reparaciones a lo largo de su vida útil, como señala la presencia de clavos pequeños de cable, más modernos y de localización acotada. El sitio de construcción de la embarcación puede haber estado cerca de las fuentes de madera, como los numerosos astilleros que existían en la ribera del río Paraná. Otra posibilidad es que los maderos hayan llegado a los astilleros de Buenos Aires, especialmente aquellos del área de La Boca, en navíos que los transportaban río abajo. Su lugar exacto de construcción es difícil de definir, pero podemos afirmar que fue manufacturada en astilleros de la cuenca del Plata y que utilizaban maderas de la región chaco-paranaense y la selva misionera.
Este caso de estudio representa una contribución al conocimiento sobre la tecnología empleada en la construcción de embarcaciones pequeñas de madera, de tradición europea, en la región de la cuenca del Plata en el siglo XIX. Esperamos que nuevas líneas de evidencia ayuden a profundizar esta cuestión y puedan ser puestas en relación con los resultados expuestos en esta tesis. Esperamos también que la metodología y las variables aquí consideradas puedan aplicarse a otras embarcaciones fragmentadas y/o descontextualizadas.

\section{Referencias citadas}

"Adams, J. (2001). Ships and boats as archaeological source material. World Archaeology, 32(3), 292-310.

"Pomey, P. (2011). Defining a Ship: Architecture, Function, and Human Space. En A. Catsambis, B. Ford y D. L. Hamilton (Eds.), The Oxford Handbook of Maritime Archaeology (pp. 25-46). Oxford: Oxford University Press.

"Schiffer, M. (2008). Behavioral Archaeology. En D. Pearsall (Ed.), Encyclopedia of Archaeology (Volumen 2) (pp. 909-919). Nueva York: Elsevier.

»Weissel, M. (1998). Informe de tareas realizadas en el dique 3 del barrio de Puerto Madero. Instituto Histórico de la Ciudad de Buenos Aires. Manuscrito inédito. 